\title{
DESENVOLVIMENTO DE BEBIDAS FERMENTADAS DE CIRIGUELA E CUPUAÇU: ESTUDO CINÉTICO, ANÁLISES CROMATOGRÁFICA E SENSORIAL.
}

\author{
L.P.S. OLIVEIRA ${ }^{1}$; D. C. L. A. CAMPELO ${ }^{1}$; I. J.CRUZ FILHO ${ }^{1}$; O. M. MARQUES ${ }^{1}$ \\ ${ }^{1}$ Universidade Federal de Pernambuco - Departamento de Engenharia Química \\ E-mail para contato: letydepaula@gmail.com
}

\begin{abstract}
RESUMO - Este trabalho teve por objetivo a formulação e desenvolvimento de bebidas fermentadas de cupuaçu e ciriguela que se apresentam como alternativa no desenvolvimento de tecnologias para a obtenção de produtos derivados com período de vida útil e valor agregado maiores. A metodologia consistiu na produção do fermentado da polpa comercial. Um reator em batelada foi utilizado sem agitação e nele adicionado o mosto do suco do fruto clarificado e o inóculo (Saccharomyces cerevisiae) a $28^{\circ} \mathrm{C}$. As análises de ${ }^{\circ}$ Brix, concentração de biomassa $\left(\mathrm{g} . \mathrm{L}^{-1}\right)$, açúcares redutores totais $\left(\mathrm{g} . \mathrm{L}^{-1}\right)$, $\mathrm{pH}$, concentração de etanol $\left(\mathrm{g} \cdot \mathrm{L}^{-1}\right)$ e cromatográficas mostraram que os vinhos estão dentro dos padrões estabelecidos pela legislação brasileira para vinhos de frutos, além também, da análise sensorial que mostrou grande aceitação, principalmente do Fermentado de Cupuaçu e seu cooler.
\end{abstract}

\section{INTRODUÇÃO}

Infelizmente em nosso país, mesmo ainda com a presença de cenários de profunda miséria e fome, nos deparamos com dados como os mostrados pelo Instituto EcoDesenvolvimento (EcoD) em sua matéria "Do campo à cidade - Soluções para o desperdício de alimentos", que diz que a cada dez toneladas de alimentos produzidos no Brasil, quatro chegam aos pratos dos brasileiros, o que representa uma montanha de 39 mil toneladas de comida que acabam no lixo, por dia. Tudo isso poderia alimentar 19 milhões de pessoas todos os dias, com as três refeições básicas: café da manhã, almoço e jantar. Em termos financeiros, o dado representa um montante de $\mathrm{R} \$ 12$ bilhões anuais jogados fora (ECOD, 2013).

No âmbito da fruticultura, temos o Brasil hoje como terceiro maior produtor mundial de frutas, com 43 milhões de toneladas ao ano (IBRAF, 2010). Infelizmente, dados mostram que o país também lidera no ranking de perdas, com dados alarmantes de cerca de 7 milhões de toneladas de frutas são jogadas fora por ano (ECOD, 2013).

Uma associação entre a quantidade produzida, a diversidade frutífera brasileira e tecnologias de inovações a favor do aproveitamento desta produção vem como incentivo no desenvolvimento de métodos de conservação que aumentem a vida útil dessas frutas.

Uma opção viável, porém, pouco explorada no nosso país com relação ao vasto potencial que apresenta, é a produção de bebidas fermentadas de frutas. $\mathrm{O}$ aumento no número de trabalhos de pesquisas relativos ao emprego de diferentes frutas na produção de bebidas alcoólicas fermentadas vem crescendo nos últimos 10 anos, todavia, o foco maior de pesquisa e desenvolvimento de tecnologias ainda tem sido direcionado a vinificação. A 
importância de se desenvolver pesquisas nesse ramo é a de necessidade de testar o procedimento técnico apropriado para cada fruta, o que requer estudos mais detalhados para determinação de metodologia apropriada (VENTURINI FILHO, 2010).

O objetivo deste trabalho foi otimizar a produção de bebidas fermentadas a partir de ciriguela e cupuaçu (frutas regionais e abundantes), estudo cinético, análises químicas e sensoriais, com o intuito de evitar o desperdício destas frutas no Brasil, gerando novos empregos e tecnologias voltadas a produção de bebidas fermentadas.

\section{MATERIAIS E MÉTODOS}

Foram utilizadas amostra comercial de polpas congeladas de ciriguela e cupuaçu da marca "Casa da Mangaba", provenientes do Centro de Abastecimento Alimentar de Pernambuco (CEASA/PE). Preparou-se o mosto da fruta, utilizando a polpa de fruta previamente descongelada e coada em filtro de pano, tanto de cupuaçu quanto de ciriguela. Verificou-se o teor de sólidos ( ${ }^{\circ}$ Brix) com a ajuda de um refratômetro. Em seguida, chaptalizou-se uma amostra de cupuaçu e outra de ciriguela com açúcar (sacarose) para fazer a correção do teor de sólidos $\left(24^{\circ}\right.$ Brix $)$ e aumentar o teor alcoólico do produto final. Com a intenção comparativa, desenvolveram-se mais duas amostras agora chaptalizadas com mel de abelha e os cálculos da diluição do mel até o ${ }^{\circ}$ Brix desejado foram feitos de acordo com o esquema recomendado por Cobenze (Meade; Chen, 1977). O mel utilizado nos experimentos foi da marca Rainha ${ }^{\circledR}$.

As formulações das amostras foram feitas de acordo com a Tabela 1.

Tabela 1 - Formulações utilizadas nas fermentações

\begin{tabular}{c|c|c}
\hline FORMULAÇÕES & MATÉRIAS-PRIMA & AGENTE DE CORREÇÃO DO ${ }^{\circ}$ BRIX \\
\hline $\mathbf{1}$ & Suco de Ciriguela & Açúcar \\
$\mathbf{2}$ & Suco de Ciriguela & Mel \\
$\mathbf{3}$ & Suco de Cupuaçu & Açúcar \\
$\mathbf{4}$ & Suco de Cupuaçu & Mel \\
\hline
\end{tabular}

$\mathrm{O}$ pH de cada mosto foi ajustado a 4,5 com carbonato de cálcio e sulfitado com 200 mg. $\mathrm{L}^{-1}$ de metabissulfito de potássio por 30 minutos. Em seguida foi inoculado com levedura comercial liofilizada (Saccharomyces cerevisiae), da marca Flesherman ${ }^{\circ}$, na concentração de $10 \mathrm{~g} . \mathrm{L}^{-1}$. A fermentação foi conduzida em quatro reatores descontínuo de $2 \mathrm{~L}$, a uma temperatura de $30^{\circ} \mathrm{C}$ até a estabilização final do ${ }^{\circ}$ Brix. Todas as amostras então foram destinadas para avaliação através de uma análise sensorial. Para avaliação sensorial os testes foram realizados com 30 julgadores não treinados constituídos de alunos, professores e funcionários da Universidade Federal de Pernambuco, com faixa etária de 18 a 30 anos de ambos os sexos. Os provadores avaliaram os atributos, cor, sabor, aroma, textura e impressão global através de um formulário com escala hedônica de nove pontos (Teixeira,1987; Cecchi, 2001). Dos fermentados de cada fruta mais aceitos pela análise sensorial, uma nova fermentação foi feita, utilizando a mesma metodologia anterior onde, desta vez, foram avaliados os parâmetros cinéticos de fermentação. O processo foi acompanhado por análises de açúcares redutores totais (ART), produto (Etanol), $\mathrm{pH}$ e acidez, durante todo o período de cultivo. A fermentação foi encerrada, quando o Brix se estabilizou. Ao final da fermentação, o produto foi mantido a $10^{\circ} \mathrm{C}$ por cinco dias, sendo em seguida filtrado, engarrafado, pasteurizado e conservado a $5^{\circ} \mathrm{C}$. Os mostos e as bebidas fermentadas foram caracterizados 
quanto ao pH, acidez titulável (IAL, 2005), açúcar redutor (AOAC,1992), biomassa e teor alcoólico (Zoecklein et al., 1994). A produtividade em etanol $\left(\operatorname{Pr}_{\mathrm{p}}\right)$ e em células $\left(\operatorname{Pr}_{\mathrm{x}}\right)$, velocidade específica máxima $\left(\mu_{\max }\right)$, o rendimento $(\mathrm{R})$ e a eficiência $\left(\varepsilon_{\mathrm{f}}\right)$ foram determinados de acordo com Schmidell et al (2001). Posteriormente, as amostras fermentadas foram destiladas, por um sistema de destilação simples, e em seguida analisadas em um Cromatógrafo CG Marca Shimadzu, modelo 17a do Laboratório de Cromatografia do Departamento de Engenharia Química da UFPE, a fim de determinar qualitativamente seus componentes voláteis.

\section{RESULTADOS E DISCUSSÃO}

As amostras, descritas pela Tabela 1, fermentaram por um tempo total de aproximadamente 90 horas e através da medição do decaimento do ${ }^{\circ}$ Brix neste tempo, pôdese observar que as amostras chaptalizadas com mel (Formulação 2 e Formulação 4) fermentaram mais rápido do que aquelas chaptalizadas com açúcar. Esta rapidez é mostrada pelas declinações das curvas destas amostras, indicando que houve consumo de açúcar logo nas primeiras horas de fermentação. A diferença evidenciada pode ser explicada pela presença de nutrientes no mel como sódio, potássio, ferro e magnésio que de forma mais significativa, beneficiaram o crescimento microbiano e a formação do produto.

$\mathrm{Na}$ análise sensorial, de acordo com a Figura 1, pode-se observar que a bebida fermentada de cupuaçu (Formulação 3) foi a que apresentou melhor aceitação por parte dos avaliadores entre as bebidas apresentadas. Em contrapartida, a bebida da mesma fruta chaptalizada com mel (Formulação 4) foi a menos aceita. Este fato pode ter ocorrido devido ao cupuaçu apresentar sabor forte e que na presença do mel de abelhas apresentou um conflito de sabor, dificultando a sua aceitação pelos julgadores. Já no caso da ciriguela, a presença do mel favoreceu a aceitação, proporcionando um aumento na média da Formulação 1 para a 2.

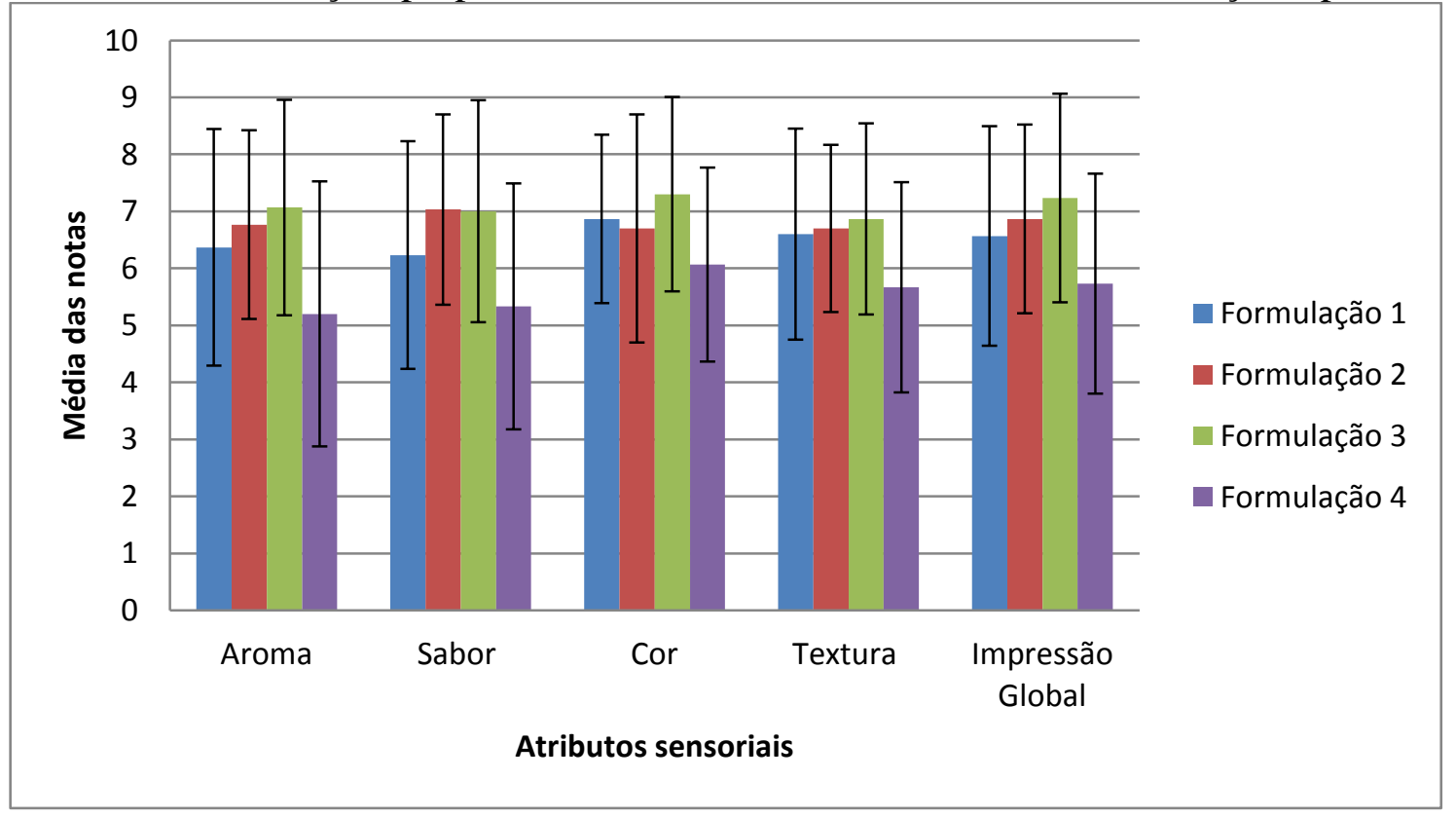

Figura 1 - Gráfico Comparativo das médias obtidas na análise sensorial em escala hedônica e escores médios e desvio padrão referente aos atributos sensoriais das bebidas alcoólicas fermentadas elaboradas. 
O teste de Tukey realizado a um nível de significância de 95\%, mostrou que a única formulação que apresentou diferença significativa das demais foi a Formulação 4 em todos os seus atributos. Os resultados do teste de Tukey encontram-se na Tabela 2.

Tabela 2 - Escores médios e desvio padrão referente aos atributos sensoriais das bebidas alcoólicas fermentadas elaboradas.

\begin{tabular}{cccccc}
\hline Formulação & Aroma & Sabor & Consistência & Aparência & Global \\
\hline $\mathbf{1}$ & $6,37^{\mathrm{ab}} \pm 2,08$ & $6,23^{\mathrm{ab}} \pm 1,99$ & $6,60^{\mathrm{ab}} \pm 1,85$ & $6,87^{\mathrm{ab}} \pm 1,48$ & $6,57^{\mathrm{ab}} \pm 1,92$ \\
$\mathbf{2}$ & $6,77^{\mathrm{a}} \pm 1,65$ & $7,03^{\mathrm{a}} \pm 1,67$ & $6,70^{\mathrm{ab}} \pm 1,47$ & $6,70^{\mathrm{ab}} \pm 2,00$ & $6,87^{\mathrm{ab}} \pm 1,66$ \\
$\mathbf{3}$ & $7,07^{\mathrm{a}} \pm 1,89$ & $7,00^{\mathrm{a}} \pm 1,95$ & $6,87^{\mathrm{a}} \pm 1,68$ & $7,30^{\mathrm{a}} \pm 1,71$ & $7,23^{\mathrm{a}} \pm 1,83$ \\
$\mathbf{4}$ & $5,20^{\mathrm{b}} \pm 2,33$ & $5,33^{\mathrm{b}} \pm 2,15$ & $5,67^{\mathrm{b}} \pm 1,84$ & $6,07^{\mathrm{b}} \pm 1,70$ & $5,73^{\mathrm{b}} \pm 1,93$ \\
\hline
\end{tabular}

Média \pm desvio padrão das análises realizadas.

*Médias seguidas de letras iguais, em uma mesma coluna, não diferem significativamente entre si, pelo teste de Tukey, a p>0,05; *Escala hedônica de nove pontos desde $9=$ gostei extremamente a $1=$ desgostei extremamente.

A cinética da fermentação das amostras de ciriguela e cupuaçu chaptalizados com sacarose foi avaliada em 172 horas de cultivo. No final do processo o açúcar residual até o final do período avaliado foi de $62 \mathrm{~g} . \mathrm{L}^{-1}$ para o cupuaçu e $45 \mathrm{~g} . \mathrm{L}^{-1}$ para ciriguela. $\mathrm{O}$ teor alcoólico elevou-se para $12,7^{\circ} \mathrm{GL}$ (concentração de $100,0 \mathrm{~g} / \mathrm{L}$ de etanol) e $14,9^{\circ} \mathrm{GL}(117,3$ $\mathrm{g} / \mathrm{L}$ ) respectivamente (Figura 2 ).
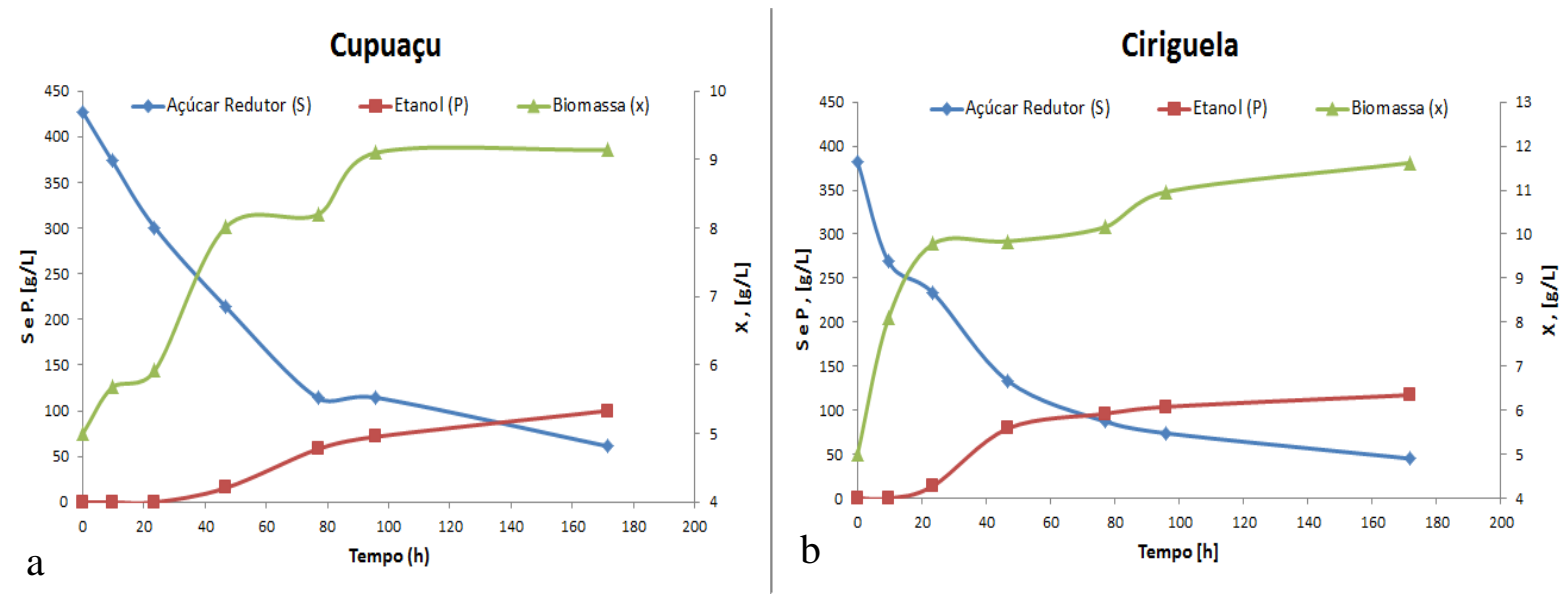

Figura 2 - Evolução cinética da biomassa (X), etanol (P) e açúcares redutores (S) ao longo da fermentação a) do Cupuaçu; b) da Ciriguela.

A eficiência e a produtividade em etanol dos fermentados de cupuaçu e ciriguela foram de $\varepsilon_{\mathrm{f}}=54$ e $68 \% ; \operatorname{Pr}_{\mathrm{p}}=0,59$ e $0,68 \mathrm{~g} \cdot \mathrm{L}^{-1} \cdot \mathrm{h}^{-1}$ respectivamente. Estes resultados foram maiores que os obtidos por Ilha e colaboradores (2008) para hidromel. A velocidade específica máxima de crescimento de $S$. cerevisiae foi $\mu_{\max }=0,023$ e $0,067 \mathrm{~h}^{-1}$ e a produtividade em célula $\operatorname{Pr}_{\mathrm{x}}=0,053$ e $0,068 \mathrm{~g} \cdot \mathrm{L}^{-1} \cdot \mathrm{h}^{-1}$ para o cupuaçu e a ciriguela, 
respectivamente. $\mathrm{O}$ pH variou de 4,72 a 4,17, enquanto que a acidez aumentou de 12 para 66mg. $\mathrm{L}^{-1}$ ao final da fermentação. A redução do $\mathrm{pH}$ decorre da produção de ácidos tais como: lático, acético e succínico (Aquaroni, 1993, Venturini Filho, 2010). A bebida produzida apresentou teor alcoólico e acidez compatíveis com os padrões de identidade e qualidade para os fermentados de frutas e que são determinantes para a estabilidade dessas bebidas.

Através da análise cromatográfica concluiu-se que os fermentados produzidos são de boa qualidade, pois, encontram-se dentro dos limites esperados para os alcoóis superiores, acetaldeídos, acetona, inclusive o perigoso metanol. Os cromatogramas são apresentados na Figura 3 e os resultados da análise cromatográfica encontram-se na Tabela 3.
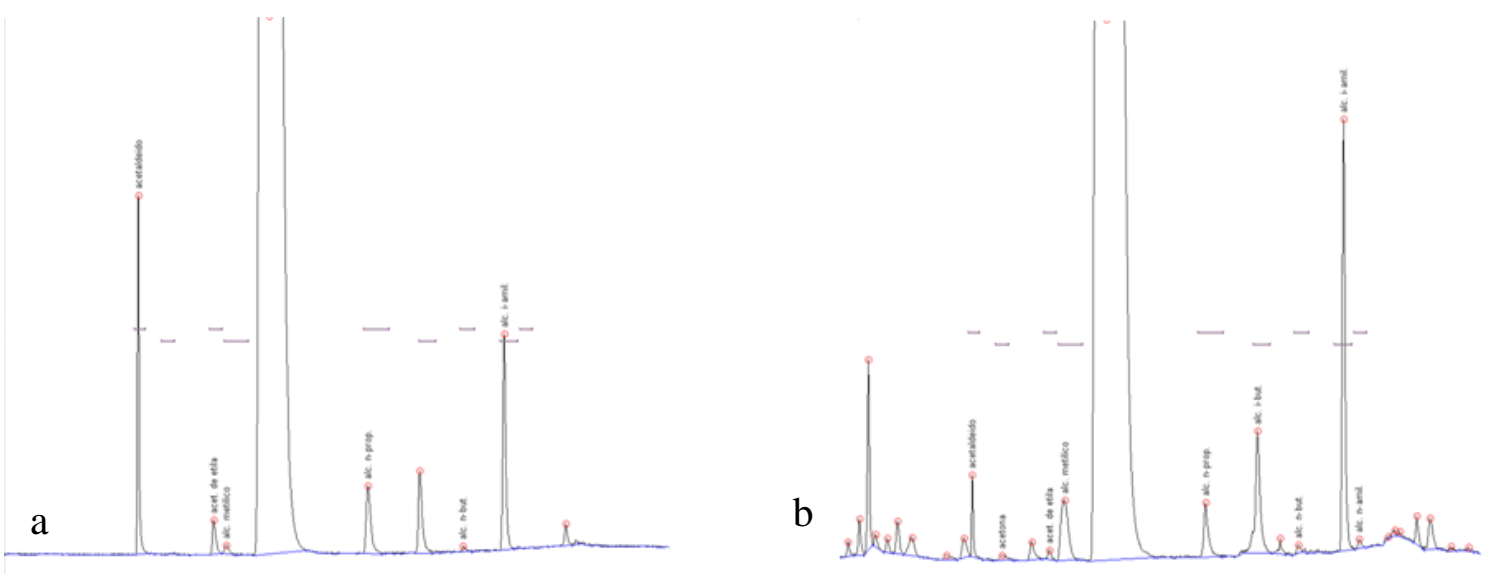

Figura 3 - Cromatogramas de Fermentado de a) Cupuaçu; b) Ciriguela.

Tabela 3 - Valores obtidos pela análise cromatográfica do Fermentado de Ciriguela e Cupuaçu

\begin{tabular}{ccc}
\hline COMPONENTE & $\begin{array}{c}\text { CIRIGUELA } \\
\text { Concentração(mg/100mL) }\end{array}$ & $\begin{array}{c}\text { CUPUAÇU } \\
\text { Concentração(mg/100mL) }\end{array}$ \\
\hline Acetaldeído & 98,02 & 409,39 \\
Acetona & 4,93 & 0,00 \\
Acetato de etila & 9,11 & 28,42 \\
Álcool metílico & 95,43 & 7,21 \\
Álcool n-propílico & 32,75 & 35,06 \\
Álcool iso-butílico & 48,88 & 0,00 \\
Álcool n-butílico & 2,50 & 1,43 \\
Álcool iso-amílico & 128,80 & 60,62 \\
Álcool n-amílico & 2,79 & 0,00 \\
\hline
\end{tabular}

De acordo com Venturini Filho (2010) concentrações totais de álcoois superiores abaixo de 400mg/L exercem um papel favorável à qualidade sensorial da bebida, no entanto teores acima disto tornam o aroma "asfixiante", depreciando a sua qualidade. A Tabela 3 
indica que o valor total obtido neste trabalho é inferior à concentração citada anteriormente, indicando que estes álcoois influenciaram positivamente o aspecto sensorial das bebidas. Foi observado também que a concentração de álcool metílico está de acordo com a Legislação Brasileira que é de, no máximo, 350mg/L (BRASIL, 1988).

A concentração total de acetaldeído está de acordo com a faixa que contribui positivamente para o aspecto sensorial citada por Venturini Filho (2010) para o Cupuaçu e próxima para a Ciriguela, de 10 a $300 \mathrm{mg} / \mathrm{L}$. Os aldeídos contribuem para formação de características de aroma e sabor e estão associados à oxidação do vinho. Assim, este é outro fator que pode ter contribuído negativamente para o aroma da graviola não ter sido tão bem aceito quanto os demais aromas.

\section{CONCLUSÃO}

Os resultados obtidos permitiram verificar que o produto obtido ficou dentro dos padrões estabelecidos pela legislação para bebidas fermentadas. A pesquisa de análise sensorial do produto foi bastante satisfatória, tendo em vista que o fermentado de cupuaçu foi o mais aceito em todos os atributos e o de ciriguela, apesar de não ser tão aceito quanto o de cupuaçu, a presença do mel na chaptalização favoreceu a bebida, aumentando sua aceitação. O processo de produção da bebida fermentada provenientes de cupuaçu e ciriguela forneceu um produto de elevado teor alcoólico, com parâmetros dentro do estabelecido e preconizado pela lei federal além de propriedades organolépticas desejáveis, sinalizando um futuro promissor neste mercado pouco explorado.

\section{REFERÊNCIAS}

AQUARONE, E.; LIMA, U. A.; BORZANI, W. Alimentos e bebidas produzidos por fermentação. Editora Edgard Blücher, São Paulo, 243 p. 1993.

ASSOCIATION OF OFFICIAL AGRICULTURAL CHEMISTS. Official methods of analysis of the Association of the Agricultural Chemists. 12 ed. Washington: A.O.A.C., 1992.

BRASIL. Lei n. 7678, de 08 de novembro de 1988. Dispõe sobre a produção, circulação e comercialização do vinho e derivados da uva e do vinho, e dá outras providências. Diário Oficial da União, Brasília, DF, 09 nov. 1988.

CECCHI, H. M. Fundamentos Teóricos e Práticos em Análise de Alimentos. Editora da UNICAMP, Campinas, São Paulo, p.212. 2001.

ECOD - Instituto EcoDesenvolvimento. Do Campo à Cidade: Soluções para o Desperdício de Alimentos. Especial Meio Ambiente, 2013.

IAL - Instituto Adolf Lutz. Métodos físico-químicos para análise de alimentos - $4^{\mathrm{a}}$ ed. 1018p. São Paulo: Instituto Adolf Lutz, 2005. 
ILHA, E. C.; TORRES, R.C.O.; BERTOLDI,F.C.; REIS,V.D.A.; ANNA, E.S. Rendimento e Eficiência da Fermentação Alcoólica na Produção de Hidromel/ Boletim de pesquisa e desenvolvimento. Corumbá MS. 2008.

INSTITUTO BASILEIRO DE FRUTAS - IBRAF. CNA discute incentivo ao consumo de frutas. 2010. Disponível em <http://www.ibraf.org.br/news/news_item.asp?NewsID=7164>. Acesso em Nov. 2011.

MEADE, G. P.; CHEN, J. C. P. Cane Sugar Handbook - 10ª ed. 811p. New York: Wiley, 1977.

SCHMIDELL, W.; LIMA, U. A.; AQUARONE, E.; BORZANI, W. 2001. Biotec. Ind: Eng. Bioq. Editora Edgard Blucher LTDA, São Paulo- SP. vol. 2. 541p.

TEIXEIRA, V. L.; PEREIRA, R. C.; JÚNIOR, A. N. M.; LEITÃO FILHO, C. M.; SILVA, C. A. R. 1987. Seasonal variations in infralitoral seaweed communities under a pollution gradient in Baía de Guanabara, Rio de Janeiro (Brazil). Ciência e Cultura 39:423-428.

VENTURINI FILHO, W. G. 2010. Bebidas alcoólicas - ciência e tecnologia, vol. 1,1 ${ }^{\mathrm{a}}$ edição, São Paulo: Blucher.

ZOECKLEIN, B.W.; FUGELSAN, K.C.; GUMP, B.H. \& NURY, F.S. Wine analysis and production. New York: Chapman \& Hall, 621p. 1994. 